\title{
The Impact of Residual Cardiovascular Risk in Combination Lipid-Modifying Therapy in the Managed Care Setting
}

\author{
Robert L. Talbert, PharmD, FCCP, BCPS, CLS
}

$\mathrm{W}$ hen considering combination lipid-modifying therapy in the managed care setting, the focus is somewhat different than an academic discussion of clinical trials and national treatment guidelines; there should be a greater emphasis on economic considerations involved with the management of cardiovascular risk. Rather than an examination of the efficacy of statins in reducing low-density lipoprotein cholesterol (LDL-C), for example, the issue becomes one of improving the cost-effectiveness of statin therapy. Taking into account both long-term medical costs and short-term pharmaceutical costs may be a difficult balancing act, but it is one that concerns managed care professionals on a daily basis. One way in which long-term medical costs could be better controlled is through the targeted use of appropriate therapeutic agents in high-risk populations. As a part of a medication therapy management program (MTMP), a medical provider statin initiation program began with the goal of investigating whether the providers, when supplied with educational materials, would prescribe statins to their untreated, high-risk patients. ${ }^{75}$ The providers for the patients included in the intervention group $(n=1,144)$ were sent educational materials and a list of their patients with coronary artery disease (CAD) or diabetes, who were not receiving statins. A control group $(\mathrm{n}=700)$ consisted of people who did not meet MTMP criteria. During the 4-month intervention period, 138 patients (12.1\%) in the intervention group started treatment with a statin versus 51 patients $(7.3 \%)$ in the control group $(P=0.001)$. Patients in the intervention group were $65 \%$ more likely (odds ratio $[\mathrm{OR}]=1.65$; $P=0.006)$ to start statin therapy than were patients in the control group. ${ }^{75}$ This study demonstrated that medical providers can be educated to consider initiating therapy. In this instance, there was an increase in the number of high-risk individuals treated with statins, which may, in the long-term, decrease incidence of costly cardiovascular events.

Treatment adherence addresses similar issues, though from a different point of view. One measure of treatment adherence is medication possession ratio (MPR), which assesses the percentage of days during which a patient has the medication. The relationship between adherence to statin therapy (MPR during a 9-month period) and achievement of LDL-C goal levels ( $\leq 100 \mathrm{mg}$ per dL) was studied in patients with diabetes or atherogenic dyslipidemia $(n=653)$ in a managed care program. ${ }^{9}$ The average MPR was significantly higher for men than for women $(0.75$ vs. $0.66 ; P<0.05)$ and, overall, 44\% ( $n=290)$ of the patients achieved an LDL-C level $<100 \mathrm{mg}$ per $\mathrm{dL}$ ( $52 \%$ of men and $37 \%$ of women; $P<0.05$ ). A significant correlation emerged between statin MPR and plasma LDL-C $(P<0.001)$, and the MPR was significantly higher in patients who achieved the LDL-C target level than in those who did not $(0.82$ vs. $0.61 ; P<0.05) .{ }^{9}$ Adherence to statin therapy, as reflected by MPR, is closely related to LDL-C goal level attainment in patients with diabetes and dyslipidemia. The probability of goal level achievement appears to increase substantially when the MPR is 0.80 (i.e., $80 \%$ ) and so that is a potentially important MPR target in managed care. Because outcomes are directly related to patients' medication-taking behavior, when clinical goals such as serum cholesterol levels are not being reached, adherence should be assessed. ${ }^{9}$

Nevertheless, even when patients adhere to statin therapy, a substantial amount of residual cardiovascular risk remains. Additionally, the residual cardiovascular risk is associated with the magnitude of LDL-C reduction. The Cholesterol Treatment Trialists' (CTT) Collaborators reported on a prospective metaanalysis of data from 90,056 individuals in 14 randomized statin trials, as described previously. In a separate analysis of the CTT data, weighted estimates of effects on different clinical outcomes per $1 \mathrm{mmol}$ per L (39 $\mathrm{mg}$ per $\mathrm{dL}$ ) reduction in LDL-C were obtained. ${ }^{1}$ During a mean follow-up of 5 years, 8,186 deaths were recorded and 14,348 individuals experienced major vascular events. There was a significant $23 \%$ proportional reduction in the incidence of first major coronary heart disease (CHD) events per $1 \mathrm{mmol}$ per L (39 $\mathrm{mg}$ per $\mathrm{dL}$ ) LDL-C reduction, in which 3,337 events occurred in patients receiving statins (7.4\% CHD event rate) and 4,420 events occurred in patients receiving placebo (9.8\% CHD event rate). ${ }^{1}$ However, significant residual cardiovascular risk remained for patients treated with statins. Addressing this residual risk, which means addressing lipid parameters beyond LDL-C, should be the new target of lipidmodifying therapy. By achieving multiple lipid levels, thereby reducing residual cardiovascular risk, a long-term reduction in cardiovascular events, and the attendant costs, may be possible.

A recent study by Alsheikh-Ali et al. examined lipid levels and the use of lipid-altering drugs in a contemporary general medical population in which patients did not have documented CHD but had CHD risk equivalents, defined by the National Cholesterol Education Program Adult Treatment Panel III (NCEP ATP III) guidelines as diabetes, peripheral artery disease (PAD), abdominal aortic aneurysm, or carotid artery disease complicated by a stroke or a transient ischemic attack $(n=877){ }^{76}$ Patients with documented CHD ( $n=635$ ) were also included in the study. On the basis of the present national guidelines, the following lipid values were considered optimal for the patents with CHD risk equivalents: LDL-C level $<100 \mathrm{mg}$ per dL; high-density lipoprotein cholesterol (HDL-C) level $\geq 40 \mathrm{mg}$ per $\mathrm{dL}$ (men) and $\geq 50 \mathrm{mg}$ per dL (women); and non-HDL-C level $<130 \mathrm{mg}$ per $\mathrm{dL}$ when triglyceride (TG) levels are $\geq 200 \mathrm{mg}$ per $\mathrm{dL} .{ }^{18}$ Of the study participants with CHD risk equivalents, most did not meet optimal lipid targets for LDL-C (67\%), HDL-C (66\%), and non-HDL-C (71\%), as shown in Figure 8. In fact, $88 \%$ of patients with CHD risk equivalents did not meet 1 or more of the guideline-established 
lipid goals. ${ }^{76}$ Statins were used by $57 \%$ of the patients, and lipidaltering drugs other than statins were rarely used in patients with CHD risk equivalents (4.7\% used niacin, $5.7 \%$ used fibrates, and $2.2 \%$ used ezetimibe). ${ }^{76}$ Specifically, in patients with low HDL-C levels ( $\mathrm{n}=577$ ), only $4.7 \%$ were taking niacin, $4.9 \%$ were taking a fibrate, and $0.9 \%$ were taking niacin and a fibrate. In patients with TG levels $\geq 200 \mathrm{mg}$ per $\mathrm{dL}$, only $9.5 \%$ were taking a fibrate, $8.2 \%$ were taking niacin, and $1.9 \%$ were taking niacin and a fibrate. This analysis highlights the dramatic need to further improve preventive measures in a substantial proportion of highrisk patients with $\mathrm{CHD}$ risk equivalents even though national guidelines urge the addition of niacin or fibrates to statins to achieve multiple lipid goals. ${ }^{18,76}$ Interestingly, the characteristics of individuals prescribed fibrates or extended-release niacin (niacin ER) differ significantly. Patients receiving initial niacin ER prescriptions were significantly older and more frequently male, had greater odds of a previous myocardial infarction (MI) diagnosis, and had greater previous use of beta-blockers and statins than did patients receiving initial fenofibrate prescriptions. ${ }^{77}$

\section{Consequences of Failing to Achieve Lipid Goals}

A significant percentage of patients do not achieve their goal levels for LDL-C or other lipid parameters. Even though the primary focus of lipid-lowering therapy should be LDL-C, approximately 50\% of those patients with known CHD do not achieve their LDL-C goal level. ${ }^{18}$ Among those with the metabolic syndrome, the percentage of patients who fail to achieve lipid goals increases to approximately $67 \%$. To address the percentage of patients who fail to achieve lipid goals, as well as the potential outcomes from this failure, longitudinal, retrospective analyses of administrative claims data and laboratory results from a U.S. managed care organization were performed in 3 patient cohorts. ${ }^{78}$ In cohort 1 , LDL-C was not optimal at baseline. In cohort 2, LDL-C plus HDL-C or TG were not optimal at baseline. In cohort 3, LDL-C, HDL-C, and TG (i.e., all 3 lipid parameters) were not optimal at baseline. The analyses revealed that there was a difference in cardiovascular event-free survival between patients who achieved optimal lipid values (OLVs) for LDL-C and those who did not (hazard ratio $[\mathrm{HR}]=0.75 ; \mathrm{P}<0.001$ ) in patient cohort $1 .{ }^{78}$ For patients in cohort 2, there was a large difference in cardiovascular event-free survival in patients who achieved OLVs for 2 lipid parameters (LDL-C and HDL-C or TG) versus those patients who did not $(\mathrm{HR}=0.54 ; \mathrm{P}<0.001){ }^{78}$ Patients in cohort 3 who achieved OLVs for all 3 lipid parameters (LDL-C, HDL-C, and TG), compared with those who did not (HR=0.54; $P=0.001$ ), showed the most striking difference in cardiovascular event-free survival. ${ }^{78}$ These data emphasize the importance of achieving OLVs for all 3 lipid parameters (LDL-C, HDL-C, and TG) to maximize the reduction in cardiovascular events. ${ }^{78}$

In a similar study, evaluating the risk of cardiovascular events, a retrospective cohort analysis was conducted using a 1.1-millionmember managed care database. ${ }^{79}$ In this analysis, the risk of
FIGURE 8 Significant Numbers of Patients Do Not Achieve Lipid Goals

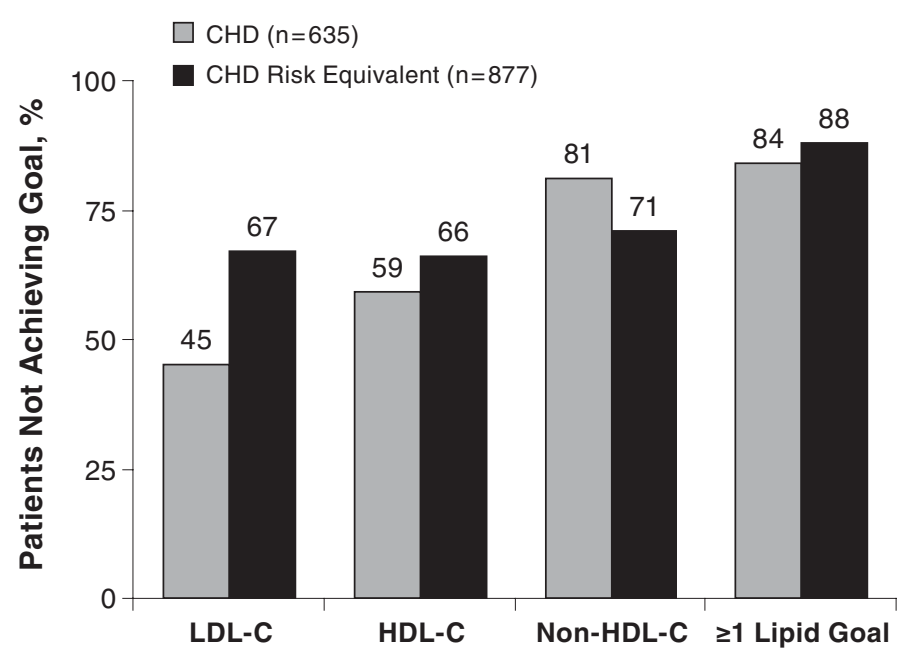

Of the 877 patients with coronary heart disease (CHD) risk equivalents $(96 \%$ had diabetes), most patients did not meet optimal lipid targets for low-desnity lipoprotein cholesterol (LDL-C) (67\%), high-density lipoprotein cholesterol (HDL-C) (66\%), and non-HDL-C (71\%). Overall, $88 \%$ of patients did not meet $\geq 1$ lipid goal (combined lipid goal). ${ }^{76}$

Lipid goals: $<100 \mathrm{mg}$ per dL LDL-C; $\geq 40 \mathrm{mg}$ per dL HDL-C (men); $\geq 50 \mathrm{mg}$ per dL HDL-C (women); $<130 \mathrm{mg}$ per dL non-HDL-C (in patients with TG $\geq 200 \mathrm{mg}$ $\operatorname{per} d L)$

Reprinted from Am J Cardiol, 2006; 98(9):1231-33: Alsheikh-Ali AA, Lin JL, Abourjaily P, Ahearn D, Kuvin JT, Karas RH. Extent to which accepted serum lipid goals are achieved in a contemporary general medical population with coronary heart disease risk equivalents. ${ }^{76}$ (with permission from Elsevier)

experiencing cardiovascular events was compared between patients who attained combined OLVs (LDL-C, HDL-C, and TG) and patients who did not attain the OLVs. The patients in the study cohort $(\mathrm{N}=30,348)$ were naive to lipid therapy, had been in the plan at least 12 months, and had a mean follow-up of 27 plus or minus 8 months. The OLVs for LDL-C, HDL-C, and TG in this study were established using the NCEP ATP III guidelines, and patients were placed into 1 of 4 groups: all 3 lipid parameters optimal, only 2 optimal, only 1 optimal, and none optimal at baseline.18,79 Although the baseline lipid values (LDL-C, HDL-C, TG, and total cholesterol) did not significantly differ among groups, patients who had experienced a cardiovascular event had a greater number of abnormal lipid values. During the follow-up period, there appeared to be only moderate improvement in lipid values and, therefore, achievement of OLVs. Stanek et al. demonstrated that the TG target was achieved most frequently and was stable over time at approximately $75 \%{ }^{79}$ Achievement of HDL-C goal levels was also frequent and stable at approximately 65\%. Thus, although TG and HDL-C targets were attained with regularity, the rate did not improve during 
FIGURE 9 Optimal Lipid Values and

Cardiovascular Event Rate

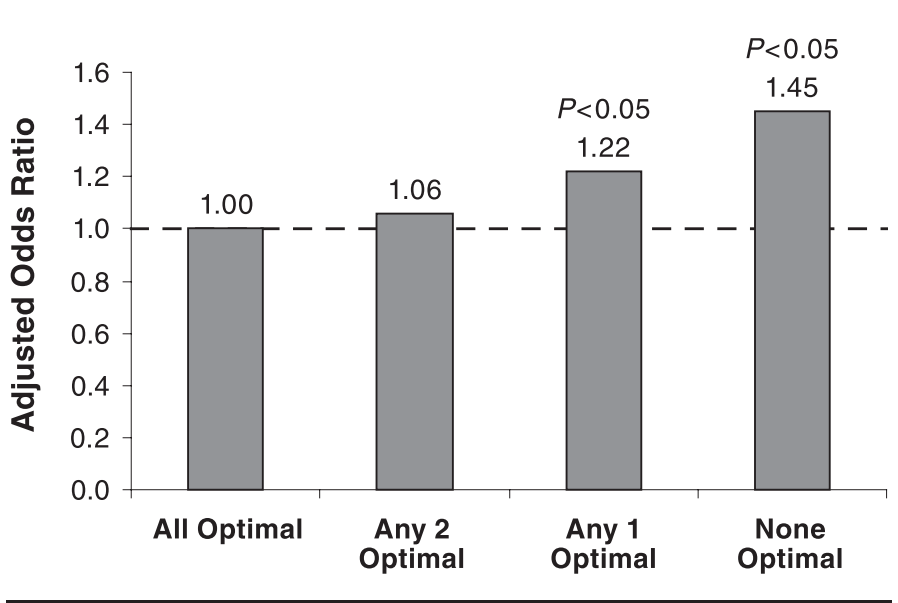

The presence of a single nonoptimal lipid value slightly increased cardiovascular $(C V)$ event risk (odds ratio $[O R]=1.06 ; 95 \%$ confidence interval $[C I]=0.95-1.18$ ), whereas 2 or all 3 nonoptimal lipid values significantly increased the risk of a CV event $(O R=1.22 ; 95 \% C I=1.08-1.37$; and $O R=1.45 ; 95 \% C I=1.24-1.68$, respectively; $P<0.05$ vs. all 3 optimal lipids using multivariate logistic regression). 79

Optimal lipid values: low-density lipoprotein cholesterol level $<130 \mathrm{mg}$ per $d L$ (elevated risk primary prevention) or $<100 \mathrm{mg}$ per $\mathrm{dL}$ (CHD), high-density lipoprotein cholesterol level $>40 \mathrm{mg}$ per $\mathrm{dL}$, and triglyceride level $<200 \mathrm{mg}$ per $\mathrm{dL}$; $n=30,348$.

follow-up. In terms of LDL-C, goal level achievement improved from approximately $30 \%$ of patients to close to $40 \%$ of patients during follow-up. Even lower than LDL-C goal achievement was attainment of combined lipid targets; during the follow-up, achievement of combined OLVs occurred in approximately 13\% of patients and increased to only approximately $20 \%$. It is apparent from these results that management of all three major lipid levels remains a challenge. ${ }^{79}$

In another analysis of these data, as shown in Figure 9, patients were placed into 1 of 4 groups at baseline: all 3 lipid parameters optimal, 2 lipid parameters optimal, 1 lipid parameter optimal, or no optimal lipid parameters. ${ }^{79}$ The definition of a cardiovascular event included diagnosis of ischemic heart disease, PAD, stroke/transient ischemic attack, or a revascularization procedure. Odds ratios (ORs) for a cardiovascular event associated with attainment of each optimal lipid parameter were determined from 5,955 cardiovascular events that occurred in 4,059 (13\%) study patients. The presence of a single nonoptimal lipid value slightly increased cardiovascular event risk (OR=1.06; $95 \%$ confidence interval $[\mathrm{CI}]=0.95-1.18$ ), whereas 2 or all 3 nonoptimal lipid values significantly increased the risk of a cardiovascular event $(\mathrm{OR}=1.22 ; 95 \% \mathrm{CI}=1.08-1.37$; and $\mathrm{OR}=1.45 ; 95 \%$ $C I=1.24-1.68$, respectively; $P<0.05$ vs. all 3 optimal lipid values). By not attaining combined OLVs, the risk of cardiovascular events was independently and significantly increased in this large at-risk population during approximately 68,283 patient-years of followup. The combination of failing to achieve optimal LDL-C with failing to achieve optimal HDL-C or TG values, or both, increased the adjusted risk of cardiovascular events by $22 \%-45 \%$. These data provide evidence for focusing therapeutic strategies on assessment and management of multiple lipid abnormalities, rather than on a single lipid abnormality. ${ }^{79}$

According to the 2008 ADA guidelines, the primary goal is an LDL-C level $<100 \mathrm{mg}$ per dL in individuals without overt cardiovascular disease, though a lower LDL-C goal $(<70 \mathrm{mg}$ per $\mathrm{dL}$ ) is an option in individuals with overt cardiovascular disease ${ }^{54}$ Additionally, the ADA recognizes serum TG concentration as a surrogate measure for atherogenic TG-rich lipoproteins and suggests a TG goal level of $<150 \mathrm{mg}$ per dL. ${ }^{54}$ Finally, the ADA standards suggest an HDL-C goal levels of $>40 \mathrm{mg}$ per dL in men and $>50 \mathrm{mg}$ per $\mathrm{dL}$ in women. Based on these guidelines, it is apparent that achieving multiple lipid targets in patients with type 2 diabetes is of great importance. In association with type 2 diabetes, medical costs increase as a function of disease severity. Patients with diabetes followed in a diabetes clinic under a managed care plan ( $n=697)$ were grouped according to severity of illness and care requirements. ${ }^{80}$ Severity of illness correlated with total medical and pharmaceutical costs. Patients were grouped according to severity of illness in 6 areas: glycemic control, cardiovascular disease, peripheral vascular disease/peripheral neuropathy, retinopathy, renal disease, and autonomic neuropathy. Patients in high- and very high-risk categories for cardiovascular disease, among other diabetes-related disease states, had markedly increased medical costs versus those patients in low-risk categories. ${ }^{80}$ Additionally, although the magnitude of the increase was much smaller and pharmaceutical costs remained reasonably similar across groups, drug costs were also significantly greater for patients in the very high-risk category. Patients who were in multiple high- and very high-risk categories had dramatically increased medical costs, as much as 10 times greater than those for patients who were in none of these categories. ${ }^{80}$ These findings indicate that people are having costly cardiovascular-related procedures, which drive the overall cost of treatment. The pharmaceutical costs, according to this study, do not increase dramatically across risk groups, and considerations beyond drug cost should be taken into account.

Studies by Cziraky et al. and Simko et al. further address attainment of lipid goals and their relationship with cardiovascular events and costs through longitudinal, retrospective analyses conducted using administrative claims data and laboratory results. ${ }^{81,82}$ Target lipid levels were established according to the NCEP ATP III guidelines, lipid goal attainment was determined in patients with and without diabetes, and the effects of lipid goal attainment on cardiovascular events and cost were determined. ${ }^{18,81,82}$ In the first analysis, a total of 10,303 patients with type 2 diabetes and 42,475 patients without type 2 diabetes were evaluated for lipid goal attainment over a mean follow-up 
of 27 plus or minus 10 months. The baseline characteristics indicated that patients with diabetes had more severe cardiovascular disease and more comorbidities, whereas patients without diabetes had a lower incidence of hypertension and CHD. ${ }^{81}$ Achievement of OLVs was analyzed during the follow-up period, which showed a fairly large difference between patients with diabetes and those without diabetes. Over the follow-up, patients with diabetes were less likely to reach combined OLVs at 12 months $(\mathrm{OR}=0.76 ; 95 \% \mathrm{CI}=0.71-0.82), 24$ months $(\mathrm{OR}=0.80 ; 95 \% \mathrm{CI}=0.75-0.85), 36$ months $(\mathrm{OR}=0.82 ; 95 \%$ $\mathrm{CI}=0.74-0.90)$, and 48 months $(\mathrm{OR}=0.77 ; 95 \% \mathrm{CI}=0.64-0.92)$ postindex event, compared with patients without diabetes. ${ }^{81}$ Thus, this retrospective analysis revealed that achievement of combined OLVs in patients with diabetes is suboptimal, which may be related to the undertreatment of all components of the lipid panel in such patients. ${ }^{81}$

Further analysis of the same database evaluated the effects of attaining multiple lipid values on cardiovascular event rates and costs. In this study, the population $(n=52,778)$ included both the diabetic and nondiabetic patients who were previously assessed separately. ${ }^{82}$ There was a significant difference in the proportion of patients who experienced ischemic heart disease or any cardiovascular event between those who achieved combined OLVs $(n=10,645)$ for LDL-C, HDL-C, and TG and those who did not $\left(n=42,133 ; P<0.05\right.$, Figure 10). ${ }^{82}$ The observed incidence of PAD and stroke between groups was not significantly different. The differences between those patients who achieve OLVs and those who do not are also reflected in medical costs. As Figure 11 shows, patients achieving combined OLVs $(n=10,645)$ for LDL-C, HDL-C, and TG showed a 9\% reduction in annual cardiovascular-related costs per patient per year, compared with patients not achieving all 3 goals $(n=42,133)(O R=0.91 ; 95 \%$ $\mathrm{CI}=0.85-0.95 ; \mathrm{P}<0.05)$. In conclusion, achieving multiple OLVs was associated with a reduced risk of cardiovascular events as well as lower associated health care costs. Adherence to lipid treatment guidelines to reduce residual risk benefits patients by reducing the risks of cardiovascular events and the health care system by lowering the overall cost. ${ }^{82}$

\section{Model-Based Analyses of Pharmacotherapy}

In these analyses, presented at various conferences, mathematical models were used to predict the effects of niacin ER in combination with simvastatin on lipid parameters in patients enrolled in managed care plans. The estimates of patients achieving lipid goals were modeled according to individual patient baseline lipid values, as determined by a full lipid panel and the current product labeling, assuming additive effects of niacin ER and simvastatin on lipid values. Therefore, the results obtained through these models express the average change that would be expected to occur. These modeling analyses were conducted on information gathered from patients selected from a 2.1-million-patient managed care database. From these records, OLV attainment

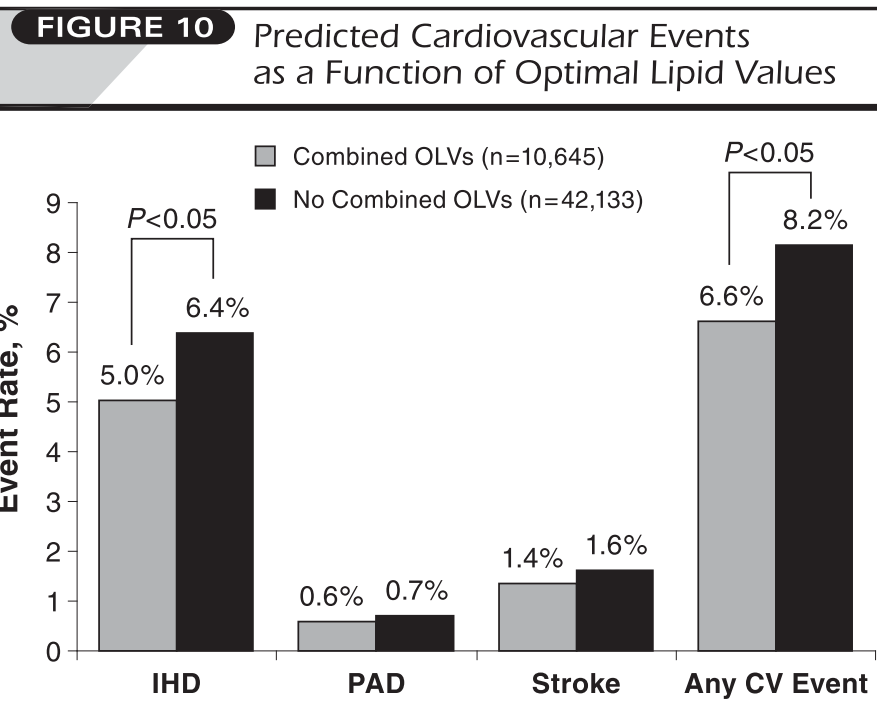

Between patients achieving combined optimal lipid values (OLVs) for low-density lipoprotein cholesterol, high-density lipoprotein cholesterol, and triglycerides $(n=10,645)$ and those who did not achieve all 3 combined OLVs $(n=42,133)$, there was a significant difference in the proportion of patients experiencing ischemic heart disease (IHD) and any cardiovascular (CV) event (multivariate analysis; $P<0.05) .82$

$n=52,778$.

$P A D=$ peripheral artery disease.

FIGURE 11 Predicted Annual Cardiovascular Costs

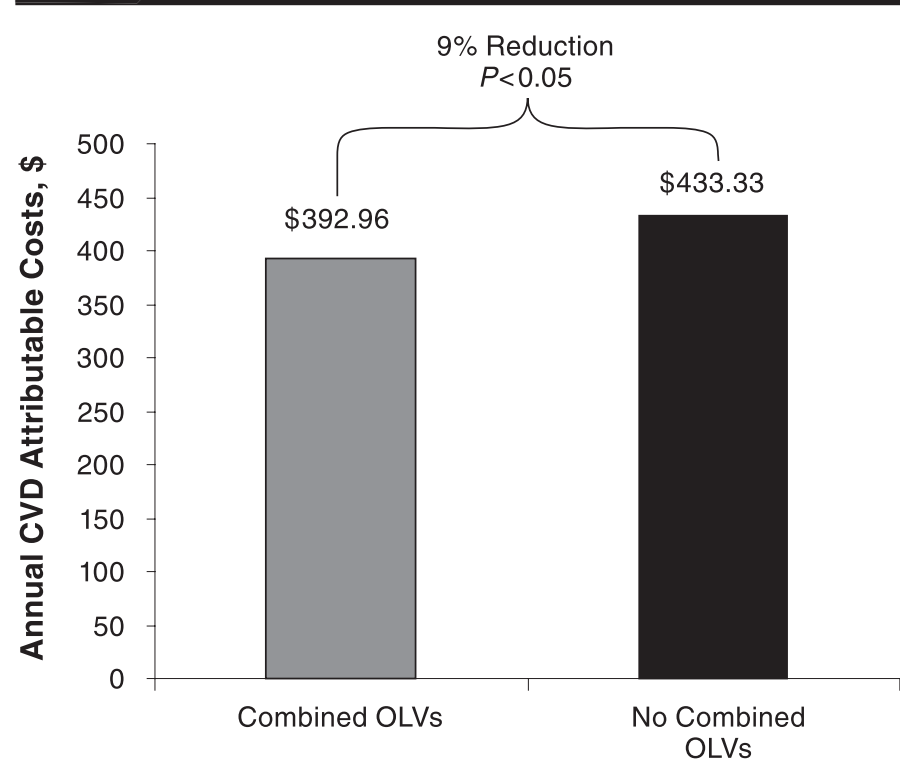

Patients achieving combined optimal lipid values (OLVs) for low-density lipoprotein cholesterol, high-density lipoprotein cholesterol, and triglycerides $(n=10,645)$ had a $9 \%$ reduction in annual cardiovascular-related costs per patient per year, compared with patients not achieving all 3 goals $(n=42,133)$ (odds ratio $=0.91$; $95 \%$ confidence interval $=0.85-0.95 ; P<0.05$ by logistic regression). 82 $C V D=$ cardiovascular disease. 


\begin{tabular}{|c|c|c|c|c|}
\hline $\begin{array}{l}\text { Lipid } \\
\text { Parameter }\end{array}$ & OLVsa & Met Syn ${ }^{b}$ & $\mathrm{DM}^{\mathrm{c}}$ & $\begin{array}{c}\text { Cardiovascular } \\
\text { Events }\end{array}$ \\
\hline DL-C & $<130 /<100^{e}$ & 131 & 125 & 131 \\
\hline HDL-C & $>40 />50$ & 43 & 47 & 48 \\
\hline Triglycerides & $<150$ & 188 & 160 & 159 \\
\hline Jon-HDL-C & $<160 /<130$ & 169 & 157 & 163 \\
\hline Total cholesterol & & 212 & 204 & 211 \\
\hline \multicolumn{5}{|c|}{$\begin{array}{l}\text { aThird Report of the National Cholesterol Education Program (NCEP) Expert } \\
\text { Panel on Detection, Evaluation, and Treatment of High Blood Cholesterol in Adults } \\
\text { (Adult Treatment Panel III) final report. Circulation. 2002;106(25):3143-21.18 } \\
\text { bStanek EJ, Quimbo RA, Cziraky MJ, Weathermon RA, Charland SL. Population- } \\
\text { based response estimates for extended-release niacin/simvastatin versus other high- } \\
\text { potency dyslipidemia therapy in patients with the metabolic syndrome. (poster) } \\
\text { Arteriosclerosis, Thrombosis, and Vascular Biology Annual Conference. Chicago, } \\
\text { IL; 2007. Abstract P438.22 } \\
\text { cStanek EJ, Charland SL, Weathermon RA, Quimbo RA, Cziraky MJ. Diabetes } \\
\text { mellitus population modeling of lipid goal achievement with extended-release } \\
\text { niacin/simvastatin versus other high-potency agents. (poster) American Diabetes } \\
\text { Association 67th Scientific Sessions. Chicago, IL; 2007. Abstract } 2445-P O .21 \\
\text { dCharland SL, Quimbo R, Cziraky MJ, Weathermon RA, Stanek EJ. Modeled } \\
\text { achievement of optimal lipid values and associated cardiovascular event rates with } \\
\text { extended-release niacin/simvastatin, ezetimibe/simvastatin, and individual agents } \\
\text { in a managed care population. (poster) Value Health. 2007;10:A56.20 } \\
\text { eAn LDL-C level } \leq 70 \text { mg per dL may be a desirable goal in patients with known } \\
\text { coronary artery disease and multiple poorly controlled risk factors. } \\
\text { Lipid values are expressed in mg per dL. } \\
\text { DM = diabetes mellitus; Met Syn=metabolic syndrome; OLV=optimal lipid value. }\end{array}$} \\
\hline
\end{tabular}

models were constructed for patients with metabolic syndrome or diabetes, and for the effects of OLV achievement on cardiovascular event rates. ${ }^{20-22}$

In the population modeling study of lipid goal achievement in metabolic syndrome patients $(n=23,773)$, effects were simulated for various lipid-modifying therapies. ${ }^{22}$ The pharmaceutical treatments compared were niacin ER/simvastatin (2,000 mg/40 mg) combination therapy, atorvastatin ( $80 \mathrm{mg}$ ), simvastatin (80 mg), simvastatin/ezetimibe ( $80 \mathrm{mg} / 10 \mathrm{mg}$ ) combination therapy, and rosuvastatin (40 mg). Table 1 shows the baseline lipid values for the patients included in the metabolic syndrome study, and the defined OLVs. ${ }^{18}$ The model revealed that, under baseline conditions (i.e., no pharmaceutical therapy), only $4 \%$ of people could be expected to achieve OLVs. ${ }^{22}$ The model further showed that atorvastatin ( $80 \mathrm{mg} ; 30.9 \%$ ), simvastatin ( $80 \mathrm{mg} ; 31.1 \%$ ), simvastatin/ezetimibe (80mg/10mg; 32.1\%), and rosuvastatin (40 mg; $39.6 \%$ ) would produce comparable effects on OLV achievement. However, when considering the niacin ER/simvastatin combination therapy, the model showed that $55.2 \%$ of patients would be expected to achieve the OLVs. The percentage with niacin ER/simvastatin was found to be significantly greater than those percentages calculated for atorvastatin, simvastatin, simvastatin/ ezetimibe, and rosuvastatin (all $P<0.05) .{ }^{22}$ According to these results, even the most potent statin produces a more than $15 \%$ lower OLV achievement than that with niacin ER/simvastatin combination therapy.

Somewhat similar results were seen in a modeling study of patients with diabetes $(n=8,164)$. Table 1 shows the baseline lipid values for the patients included in this model. As in the metabolic syndrome model, the model treatments were niacin ER/ simvastatin $(2,000 \mathrm{mg} / 40 \mathrm{mg})$ combination therapy, atorvastatin (80 mg), simvastatin (80 mg), simvastatin/ezetimibe (80 mg/10 $\mathrm{mg}$ ) combination therapy, and rosuvastatin (40 mg). ${ }^{21}$ The model showed that, at baseline (no pharmacological therapy), only $7.4 \%$ of patients with diabetes would be expected to meet all OLVs. In this patient population, all of the potential drug treatments were found to be more effective in attaining OLVs than in metabolic syndrome patients. Again, the modeled percentage of patients achieving OLVs with niacin ER/simvastatin combination therapy (62.8\%) was significantly greater $(P<0.05$ for all between treatment comparisons) than the percentages calculated with atorvastatin (45.4\%), simvastatin (41.9\%), simvastatin/ezetimibe (45.9\%), or rosuvastatin (52.3\%). In patients with type 2 diabetes, the niacin ER/simvastatin combination therapy was projected to result in $10 \%$ more patients attaining OLVs than with rosuvastatin. ${ }^{21}$

The final population modeling study of interest in this discussion examined the achievement of OLVs and cardiovascular event reduction as a consequence of various pharmacologic interventions, including niacin ER/simvastatin and simvastatin/ ezetimibe, as well as niacin ER, simvastatin, and ezetimibe monotherapies. ${ }^{20}$ In this model, cardiovascular risk and cardiovascular events were identified for each patient $(n=44,351)$ and the population cardiovascular event rate during the follow-up (30 plus or minus 12 months) was calculated to be $15.2 \%$ $(\mathrm{OR}=0.69 ; \mathrm{CI}=0.61-0.81)$, which places these patients at high risk according to Framingham score prediction. Achievement of OLVs (18\% of patients) versus nonachievement of OLVs (82\%) was used to estimate the cardiovascular event rates associated with OLV achievement. ${ }^{20}$ Table 1 shows baseline lipid values and OLVs. ${ }^{20}$ Figures 12 and 13 show the results from these models. Figure 12 shows the hypothetical achievement of OLVs with the different treatments. In this model, as in the models described previously, niacin ER/simvastatin combination therapy yielded a significantly greater percentage of patients (66.2\%) who could be expected to achieve OLVs, compared with other treatments in the model $(P<0.05$ compared with simvastatin/ezetimibe, niacin ER, and simvastatin) due to the additive beneficial effects expected with the combination of niacin with the statin. ${ }^{20}$ Additionally, ezetimibe monotherapy was expected to result in a much lower percentage of patients achieving OLVs than the other treatments. In several trials, ezetimibe has been demonstrated to reduce LDL-C levels by approximately 18\%. Compared with statins, ezetimibe is less potent and has little or no effect on either TG or HDL-C levels, so it is not surprising that OLV attainment is likely to be lower under this therapeutic condition. ${ }^{20}$ Figure 13 shows the relative risk reductions associated with the different therapies. 
Under baseline conditions the cardiovascular event rate is estimated to be $15.2 \%$, whereas a relative risk reduction is observed across all of the interventions studied. The largest risk reduction (38\%) occurred under the niacin ER/simvastatin treatment condition, in which the $15.2 \%$ baseline cardiovascular event rate was reduced to $9.4 \%$, which was significantly different from the cardiovascular event reductions calculated for the other treatment conditions $(P<0.05$ vs. simvastatin/ezetimibe, niacin ER, and simvastatin).

A separate modeling study examined the effects of niacin ER/ simvastatin combination therapy on the medical costs of CHD events. These medical costs during a 5 -year period were projected based on data gathered from clinical trials, national databases, and published literature, and took into account emergency, inpatient, and outpatient costs. ${ }^{23}$ These models were based on hypothetical cohorts of 10,000 patients with CHD who had any abnormal lipid values for LDL-C, HDL-C, TG, and non-HDL-C. Therapeutic agents in the models included simvastatin monotherapy at various doses or fixed-dose combination (FDC) niacin ER/ simvastatin. Direct medical costs were expressed in dollars with an annual discount rate of $3 \% .{ }^{23}$ The modeled medical costs are shown in Table 2. Compared with simvastatin monotherapy (20 mg), the low-dose niacin ER/simvastatin combination therapy $(1,000 \mathrm{mg} / 20 \mathrm{mg})$ yields an $8.8 \%$ reduction in costs. At the highest dose of niacin ER/simvastatin $(2,000 \mathrm{mg} / 40 \mathrm{mg}$ ) combination therapy, an $11 \%$ reduction in cost was calculated compared with simvastatin monotherapy $(80 \mathrm{mg})$. Therefore, in terms of pharmaceutical costs, niacin ER/simvastatin FDC results in a noteworthy cost savings. ${ }^{23}$

\section{Fixed-Dose Niacin ER/Simvastatin}

\section{Combination Therapy}

For many chronic conditions, such as hypertension, dyslipidemia, and diabetes, adequate therapy is typically not achieved through the use of just one drug. Instead, multiple drug therapies must often be used to target multiple risk factors or disease-associated complications. For example, patients with diabetes must often be managed through a combination of lipid-lowering therapies, antihypertensives, and hypoglycemic medications; as the number of drugs required increases, so does the treatment cost..$^{24}$ Therefore, potentially beneficial methods to address drug number include the combination of 2 or more drugs in a single capsule or tablet and the development of individual drugs that address multiple risk factors. Both of these approaches should reduce pharmaceutical costs. ${ }^{24}$ Using FDC therapy reduces the number of pills (i.e., pill burden), simplifies the dosing regimen, and ensures that the correct dosage of each component is taken. ${ }^{83,84}$ FDC therapy also improves medication compliance, which is often associated with enhanced outcomes. ${ }^{83,84}$

Evidence for benefits conferred by a FDC therapy has been reported from a prescription database analysis that examined patients $(n=6,206)$ taking the same 2 antihypertensive drugs

\section{FIGURE 12 Modeled Achievement of OLVs}

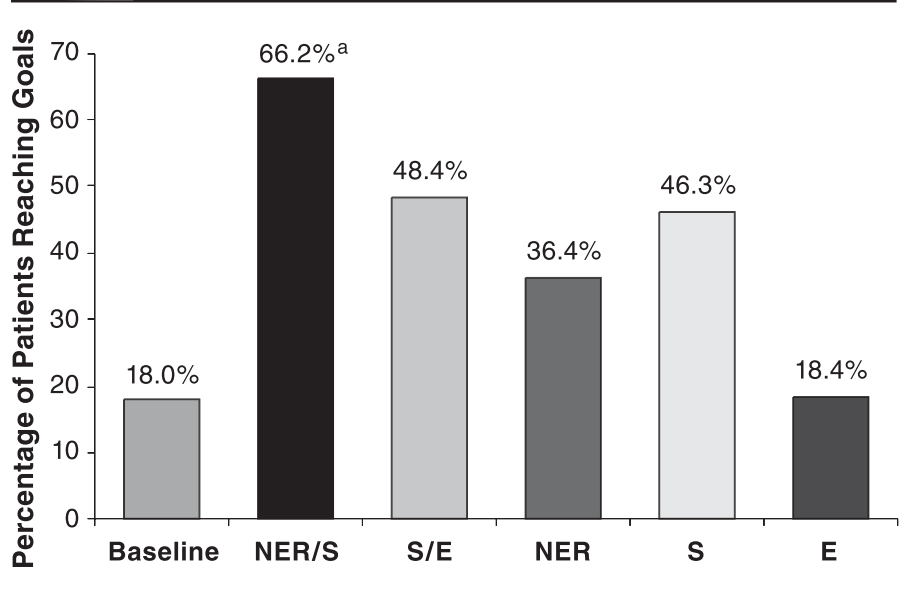

Optimal lipid values (OLVS) are defined as low-density lipoprotein cholesterol level $<130 / 100 \mathrm{mg}$ per dL per risk level, high-density lipoprotein cholesterol $>40 \mathrm{mg}$ per $d L$ in men (50 mg per dL in women), triglyceride level $<150 \mathrm{mg}$ per $d L$, and non-HDL-C level $<160 / 130 \mathrm{mg}$ per $\mathrm{dL}$ per risk level. The percentage of patients predicted to achieve OLVs with extended-release niacin (NER)/simvastatin (S) combination therapy was significantly greater than those percentages for other treatments

a $P<0.05$ versus $S / E, N E R$, and $S$ by chi-square; $N=44,351$.

$E=$ ezetimibe.

\section{FIGURE 13 Modeled Cardiovascular Event Rate Reduction ${ }^{a}$}

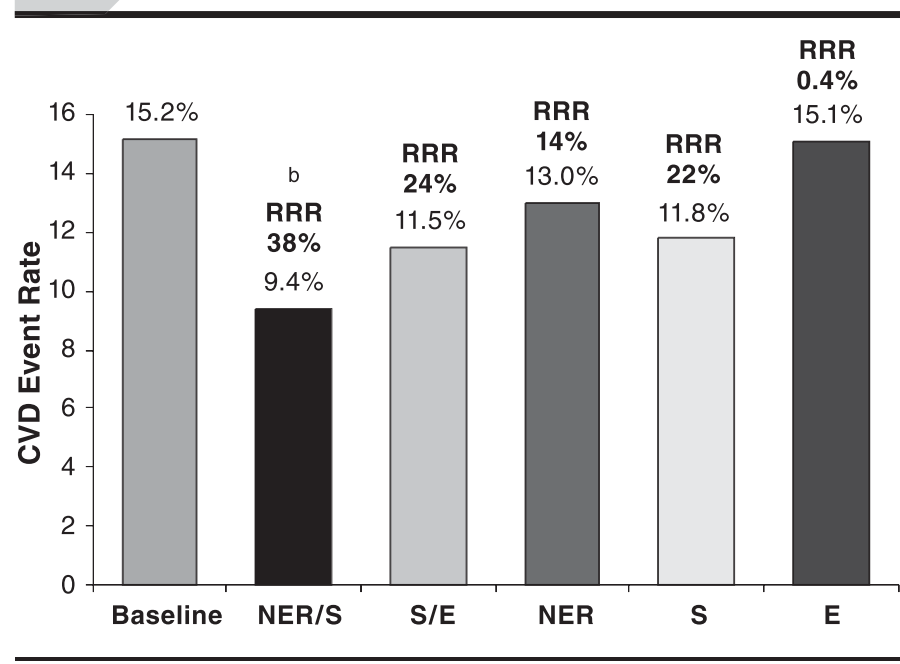

The relative risk reduction with extended-release niacin (NER)/simvastatin (S) combination therapy was predicted to be significantly greater than for other treatments

a Charland SL, Quimbo R, Cziraky MJ, Weathermon RA, Stanek EJ. Modeled achievement of optimal lipid values and associated cardiovascular event rates with extended-release niacin/simvastatin, ezetimibe/simvastatin, and individual agents in a managed care population. (poster) Value Health. 2007;10:A56.20

${ }^{b} P<0.05$, compared with Slezetimibe (E), NER, and S by chi-square; $n=44,351$. $C V D=$ cardiovascular disease; $R R R=$ relative risk reduction versus baseline $C V D$ event rate. 


\section{TABLE 2 Reduced Costs With} Niacin ER/Simvastatin ${ }^{a}$

\begin{tabular}{c|c}
\hline Treatment Comparisons & Cost Reduction With NER/S \\
\hline S20 vs. NER/S $(1,000 \mathrm{mg} / 20 \mathrm{mg})$ & $8.8 \%$ \\
\hline $\mathrm{S} 40 \mathrm{vs} . \mathrm{NER} / \mathrm{S}(1,000 \mathrm{mg} / 40 \mathrm{mg})$ & $9.1 \%$ \\
\hline $\mathrm{S} 80 \mathrm{vs} . \mathrm{NER} / \mathrm{S}(2,000 \mathrm{mg} / 40 \mathrm{mg})$ & $11.2 \%$ \\
\hline
\end{tabular}

airect medical costs of coronary heart disease events, expressed in \$U.S. 2006, over 5 years.

$E R=$ extended release; $N E R=$ niacin $E R ; S=$ simvastatin .

\section{FIGURE 14 Fixed-Dose Combination Therapy Improves Adherence Rates}

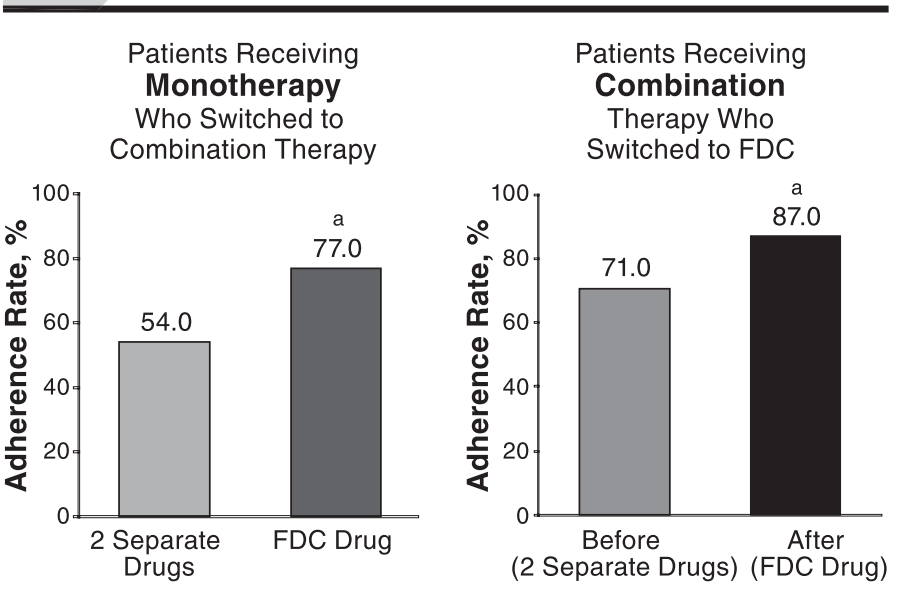

As shown on the left, in the patients receiving monotherapy $(n=1,815)$ who required the addition of the alternative agent, resulting in combination therapy with 2 separate drugs, adherence rates were significantly lower (54.0\%) than in the patients receiving monotherapy $(n=105)$ who were switched to fixed-dose combination (FDC) therapy $(77.0 \% ; P<0.001$ vs. combination therapy with 2 separate drugs by analysis of covariance [ANCOVA]). ${ }^{86}$ As shown on the right, patients receiving combination therapy as 2 separate drugs who were switched to FDC therapy $(n=59)$ had a significant improvement in adherence after the switch (71.0\% vs. 87.0\%; $P<0.001$ by ANCOVA).

a $P<0.001$ versus 2 separate drugs.

individually or as a FDC. Adherence patterns of patients were studied through a retrospective analysis of pharmacy claims from a managed care organization in the northeastern United States. ${ }^{85}$ Overall, MPRs were significantly higher for the FDC than for the 2 separate drugs ( $88 \%$ vs. $69 \%, P<0.001$ ), regardless of the number of concomitant drugs prescribed. ${ }^{85}$ In addition, adherence rates were significantly higher in the FDC group during all 3-month assessment periods ( $P<0.001$ for all comparisons). At 3 months, MPR was increased by approximately 10\%; at 12 months, there was an MPR difference of approximately 20\%. Thus, patients prescribed a FDC drug had possession of their medication for a significantly greater percentage of time over the 1-year follow-up period than did patients prescribed 2 drugs taken separately. As the number of concomitant drugs increased, the difference in the MPR between the 2 treatment groups increased in favor of the FDC drug, which suggests that it would be advantageous to use FDCs more frequently. ${ }^{85}$

A similar study analyzed the effect of switching between separate drug therapies and FDC therapies on medication adherence among patients with diabetes in a managed care organization. Medication adherence, defined as the sum of the days' supply of medication obtained by the patient during the follow-up period divided by the total number of days in the designated follow-up period (180 days), was evaluated through a retrospective database analysis of pharmacy claims. After adjustment for potential confounding factors, including overall medication burden at index, there were no significant differences in adherence rates in 6,502 newly treated patients receiving monotherapy, combination therapy, or FDC therapy. ${ }^{86}$ However, as shown in Figure 14 (left panel), among the 1,815 previously treated patients receiving monotherapy who required the addition of the alternative agent, resulting in combination therapy with 2 separate drugs, adherence rates were significantly lower $(54.0 \%)$ than in the 105 patients receiving monotherapy who were switched to FDC therapy $(77.0 \% ; P<0.001$ vs. combination therapy with 2 separate drugs). As shown in Figure 14 (right panel), the 59 previously treated patients receiving combination therapy as 2 separate drugs who were switched to FDC therapy showed a significant improvement in adherence after the switch $(71.0 \%$ vs. $87.0 \% ; P<0.001) .86$ Thus, previously treated patients receiving monotherapy who then required additional therapy showed significantly greater adherence when they were switched to FDC therapy, compared with combination therapy with 2 separate drugs. Furthermore, patients receiving combination therapy with 2 separate drugs who were switched to FDC therapy showed significantly greater medication adherence after the switch. ${ }^{86}$

The Safety and Efficacy of Fixed Dose Niacin ER and Simvastatin Combination Therapy (SEACOAST) trial, which investigated niacin ER/simvastatin FDC therapy, has been recently published. ${ }^{25,26}$ This 24 -week randomized clinical trial compared simvastatin monotherapy with a novel combination of niacin ER/simvastatin in patients with mixed dyslipidemia, as identified by elevated levels of non-HDL-C. ${ }^{25,26}$ After a simvastatin run-in phase during which patients received either 20 $\mathrm{mg}$ or $40 \mathrm{mg}$ of simvastatin for at least 2 weeks, patients were assigned to either simvastatin low-dose groups (SEACOAST I) or simvastatin high-dose groups (SEACOAST II), which were determined by the final run-in simvastatin dose. Patients were then randomized to 1 of 3 treatment groups in each half of the study. In SEACOAST I, the treatment groups were simvastatin $(20 \mathrm{mg})$, niacin ER/simvastatin ( $1,000 \mathrm{mg} / 20 \mathrm{mg}$ ), and niacin ER/ simvastatin $(2,000 \mathrm{mg} / 20 \mathrm{mg}){ }^{26}$ In SEACOAST II, which included more intensive lipid-modifying therapy during both the run-in phase and the 24-week trial phase, the treatment groups were simvastatin $(80 \mathrm{mg}$ ), niacin ER/simvastatin (1,000 mg/ 


\section{FIGURE 15 SEACOAST I: Efficacy of Niacin ER/Simvastatin Combination Therapy}

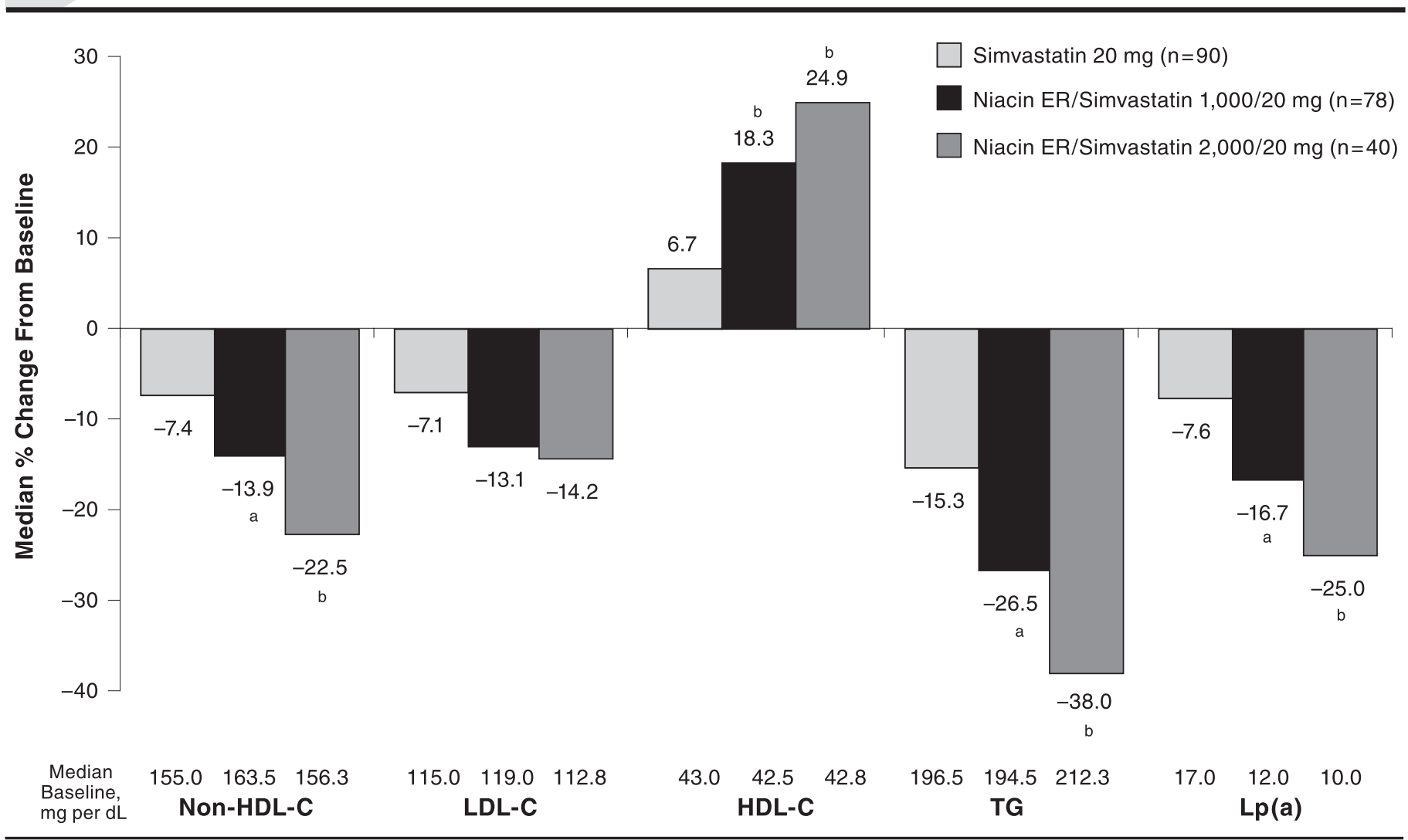

Extended-release niacin (niacin ER)/simvastatin (1,000 mg/20 mg and 2,000 mg/20 mg) fixed-dose combination (FDC) therapies demonstrated significant, dose-related improvements in non-high-density lipoprotein cholesterol (non-HDL-C), high-density lipoprotein cholesterol (HDL-C), triglycerides (TG), and lipoprotein A (Lp[a]), compared with simvastatin $(20 \mathrm{mg}$ ) monotherapy, as revealed by nonparametric analyses. 26

a $P<0.05$ versus simvastatin $(20 \mathrm{mg})$.

$\mathrm{b} P<0.001$ versus simvastatin $(20 \mathrm{mg})$.

$40 \mathrm{mg}$ ), and niacin ER/simvastatin (2,000 mg/40 mg). The primary end point in SEACOAST was the median percent change in non-HDL-C during the period from baseline to week 24, beginning from the end of the simvastatin run-in. ${ }^{25,26}$

In SEACOAST I, the niacin ER/simvastatin (1,000 mg/ $20 \mathrm{mg}$ and 2,000 mg/20 mg) FDC therapies produced significant, dose-related improvements in non-HDL-C, HDL-C, TG, and lipoprotein A (Lp[a]), compared with simvastatin (20 mg) monotherapy. ${ }^{26}$ Figure 15 shows a $22.5 \%$ reduction in non-HDLC levels with high-dose niacin ER/simvastatin versus a $7.4 \%$ reduction with simvastatin monotherapy. Although there was a dose-related response in LDL-C level reduction, there were no significant differences between groups in this lipid parameter; simvastatin alone resulted in a $7.1 \%$ decrease in LDL-C levels, whereas high-dose niacin ER/simvastatin FDC therapy produced a $14.2 \%$ reduction. Perhaps the most interesting result was the 24.9\% increase in HDL-C associated with high-dose niacin ER/ simvastatin. The FDC therapy would appear to confer a much greater benefit than most statin monotherapies, which provides further support for considering combination therapy to achieve multiple lipid targets. The results of SEACOAST I also showed substantial reduction of TG that, because of the niacin component of the FDC therapy, reached 38\% in the high-dose group. Statins tend to have a TG-lowering effect, but this effect is generally proportional to the size of LDL-C reduction and depends on the baseline TG concentration and the dose or potency of the statin used. Finally, a substantial $25.0 \%$ reduction in Lp(a) was observed with the high-dose niacin ER/simvastatin treatment, whereas little change in $\operatorname{Lp}(\mathrm{a})$ was observed with simvastatin monotherapy, which is consistent with previous reports in the literature. $\mathrm{Lp}(\mathrm{a})$ is a lipoprotein particle that is not routinely measured, but is considered by many to be as atherogenic as LDL-C.

A similar pattern of results was seen in SEACOAST II, in which the primary end point was the median percent change from 


\section{FIGURE 16 SEACOAST II: Efficacy of Niacin ER/Simvastatin Combination Therapy}

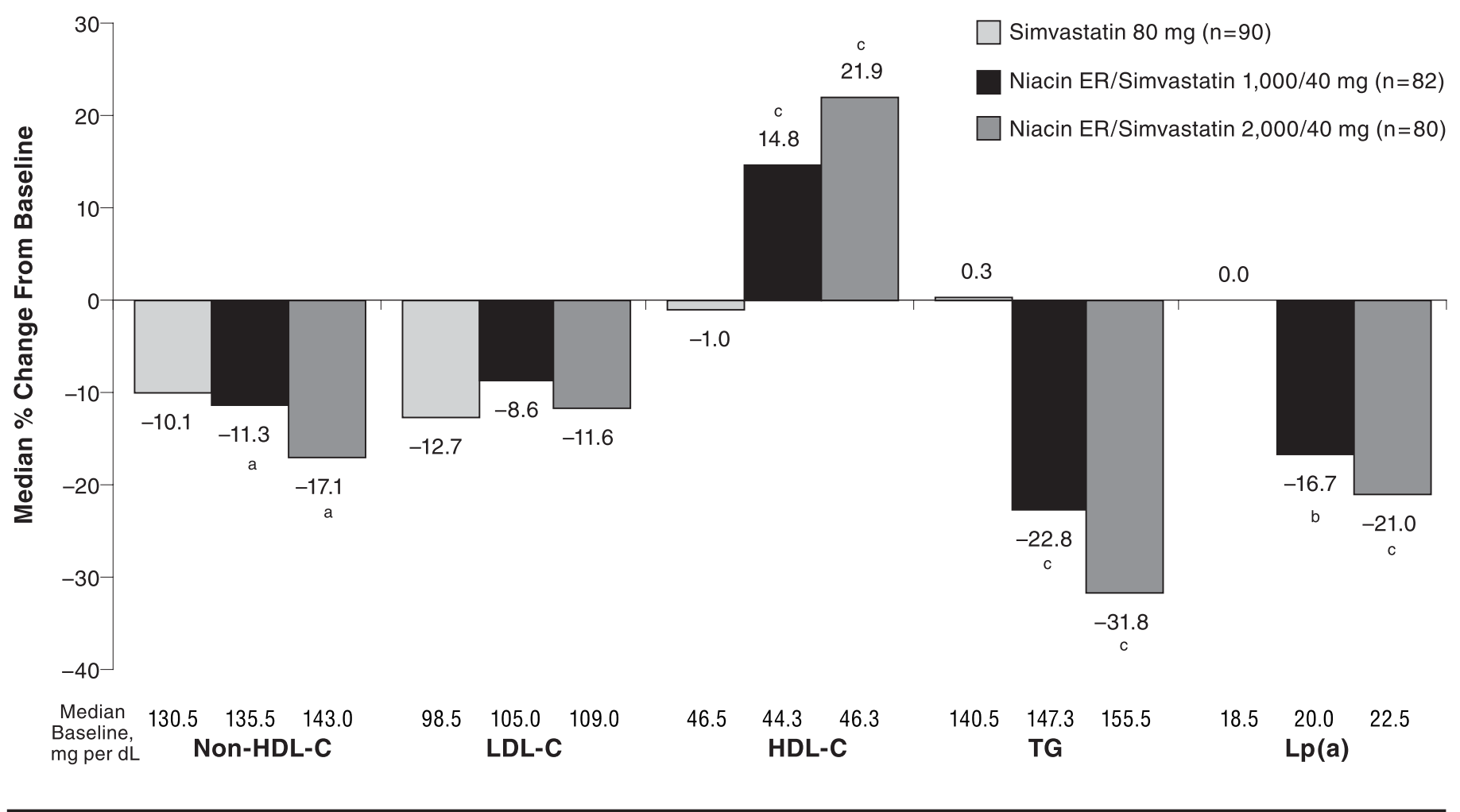

Extended-release niacin (niacin ER)/simvastatin (1,000 $\mathrm{mg} / 40 \mathrm{mg}$ and 2,000 $\mathrm{mg} / 40 \mathrm{mg}$ ) fixed-dose combination (FDC) therapies were comparable to simvastatin ( $80 \mathrm{mg}$ ) monotherapy (double the simvastatin dose) in reduction of non-high-density lipoprotein cholesterol (non-HDL-C) levels. Both niacin ER/simvastatin (1,000 mg/40 mg and $2,000 \mathrm{mg} / 40 \mathrm{mg}$ ) FDC therapies resulted in significant, dose-related improvements in HDL-C, lipoprotein A (Lp [a]), and triglycerides (TG), compared with simvastatin $(80 \mathrm{mg}$ ) therapy (double the simvastatin dose), as revealed by nonparametric analyses. 25 Simvastatin $40 \mathrm{mg}$ at baseline.

${ }^{a}$ Comparable to simvastatin $(80 \mathrm{mg})$.

b $P<0.01$ versus simvastatin $(80 \mathrm{mg})$.

c $P<0.001$ versus simvastatin $(80 \mathrm{mg})$.

baseline to week 24 in non-HDL-C (from a simvastatin [40 mg] run-in baseline). Figure 16 shows that the changes may appear to be somewhat more modest than those in SEACOAST I; however, the patients in SEACOAST II received more intensive statin therapy during the run-in phase of the study. A $17.1 \%$ reduction in the primary end point of non-HDL-C was seen with the high-dose niacin ER/simvastatin $(2,000 \mathrm{mg} / 40 \mathrm{mg})$ FDC therapy versus a $10.1 \%$ reduction with simvastatin $(80 \mathrm{mg})$ monotherapy, although this difference was not statistically significant. Decreases in levels of LDL-C were observed to be comparable across all treatment groups. Both fixed-dose niacin ER/simvastatin $(1,000 \mathrm{mg} / 40 \mathrm{mg}$ and $2,000 \mathrm{mg} / 40 \mathrm{mg}$ ) combination therapies resulted in significant, dose-related improvements in HDL-C, Lp(a), and TG levels, compared with simvastatin $\left(80 \mathrm{mg}\right.$ ) therapy. ${ }^{25}$ Across these lipid parameters, simvastatin $80 \mathrm{mg}$ monotherapy had essentially no effect beyond that conferred by the $40 \mathrm{mg}$ dose of simvastatin administered during the run-in phase.

To summarize the SEACOAST trial, niacin ER/simvastatin FDC therapy yielded significant improvements in levels of atherogenic (non-HDL-C, TG, and Lp[a]) and antiatherogenic (HDL-C) particles, compared with simvastatin monotherapy over a 24-week period. Treatment with niacin ER/simvastatin for 24 weeks was well tolerated with no unanticipated adverse effects. Only flushing/pruritus was significantly more frequent with niacin ER/simvastatin FDC therapy, compared with simvastatin monotherapy. There was no evidence of increased risk of hepatotoxicity or myopathy with niacin ER/simvastatin therapy. Overall, these data support the safe and efficacious use of niacin ER/ simvastatin FDC therapy in a broad population of dyslipidemic patients to help them reach and maintain multiple OLVs. ${ }^{25,26}$ 


\section{Summary}

Statins are often the initial pharmaceutical intervention for reducing LDL-C levels and cardiovascular event risk, and usage of statins in managed care populations may help to reduce the long-term costs associated with cardiovascular events. However, as demonstrated in the first article, residual cardiovascular risk remains after statin therapy, which can be linked to elevated levels of TG and non-HDL-C as well as low HDL-C. Studies modeling attainment of multiple lipid targets in managed care populations have demonstrated that the achievement of combined OLVs, rather than simply achieving a goal for LDL-C reduction, is predicted to be associated with a reduced risk of cardiovascular events and lower associated health care costs. Modeling studies also estimated that OLVs would be more frequently achieved in managed care organization populations with niacin ER/simvastatin FDC therapy than with other highpotency agents and, subsequently, would provide significant reductions in projected cardiovascular event rates. Moreover, models indicated that lipid-modifying therapy with a niacin ER/ simvastatin combination would be expected to reduce direct medical costs of CHD events more effectively than high-dose simvastatin monotherapy. Because one of the important factors in any treatment is medication adherence, FDC therapy may prove to be highly beneficial in treating multiple lipid abnormalities. Indeed, the SEACOAST clinical trial showed that a niacin ER/simvastatin FDC therapy produced significantly greater effects on various lipid parameters than did simvastatin monotherapy. 